\title{
Modeling and energy efficiency optimization of belt conveyors
}

\author{
Shirong Zhang ${ }^{\mathrm{a}, *}$, Xiaohua Xia ${ }^{\mathrm{b}}$ \\ ${ }^{a}$ Department of Automation, Wuhan University, Wuhan 430072, China \\ ${ }^{\mathrm{b}}$ Department of Electrical, Electronic and Computer Engineering, University of Pretoria, Pretoria 0002, South Africa
}

\section{A R T I C L E I N F O}

\section{Article history:}

Received 14 October 2010

Received in revised form 3 March 2011

Accepted 16 March 2011

Available online $\mathrm{xxxx}$

\section{Keywords:}

Analytical energy model

Belt conveyor

Operation efficiency optimization

Parameter estimation

\begin{abstract}
A B S T R A C T
The improvement of the energy efficiency of belt conveyor systems can be achieved at equipment and operation levels. Specifically, variable speed control, an equipment level intervention, is recommended to improve operation efficiency of belt conveyors. However, the current implementations mostly focus on lower level control loops without operational considerations at the system level. This paper intends to take a model based optimization approach to improve the efficiency of belt conveyors at the operational level. An analytical energy model, originating from ISO 5048, is firstly proposed, which lumps all the parameters into four coefficients. Subsequently, both an off-line and an on-line parameter estimation schemes are applied to identify the new energy model, respectively. Simulation results are presented for the estimates of the four coefficients. Finally, optimization is done to achieve the best operation efficiency of belt conveyors under various constraints. Six optimization problems of a typical belt conveyor system are formulated, respectively, with solutions in simulation for a case study.
\end{abstract}

(c) 2011 Elsevier Ltd. All rights reserved.

\section{Introduction}

Material handling is an important sector of industry, which is consuming a considerable proportion of the total power supply. For instance, material handling contributes about $10 \%$ of the total maximum demand in South Africa [1]. Belt conveyors are being employed to form the most important parts of material handling systems because of their high efficiency of transportation. It is significant to reduce the energy consumption or energy cost of material handling sector. This task accordingly depends on the improvement of the energy efficiency of belt conveyors, for they are the main energy consuming components of material handling systems. Consequently, energy efficiency becomes one of the development focuses of the belt conveyor technology [2].

A belt conveyor is a typical energy conversion system from electrical energy to mechanical energy. Its energy efficiency can generally be improved at four levels: performance, operation, equipment, and technology [3]. However, the majority of the technical literature concerning the energy efficiency of belt conveyors focus on the operational level and the equipment level.

In practice, the improvement of equipment efficiency of belt conveyors is achieved mainly by introducing highly efficient equipment. The idler, belt and drive system are the main targets. In [4], the influences on idlers from design, assembly, lubrication, bearing

\footnotetext{
* Corresponding author. Tel.: +86 276877 2169; fax: +86 2768772272.

E-mail address: srzhang@whu.edu.cn (S. Zhang).
}

seals, and maintenance are reviewed. Energy saving idlers are proposed and tested in $[5,6]$. Energy optimized belts are developed in [7] by improving the structure and rubber compounds of the belts. Energy-efficient motors, and variable speed drives (VSDs) are recommended in [8]. In general, extra investment is needed for the equipment retrofitting or replacement; and the efficiency improvement opportunities are limited to certain equipment.

Operation is another aspect for energy efficiency of belt conveyors. In [9-11], the operation efficiency in terms of operational cost of belt conveyors is improved by introducing load shifting. Speed control is recommended for energy efficiency of belt conveyor systems, even though it is occasionally challenged, e.g., in [12]. The core of speed control is to keep a constantly high amount of material along the whole belt, which is believed to have high operation efficiency. The theoretical analysis along with experimental validation on a VSD based conveying system is presented in [14]. Nowadays, the idea of speed control has been adopted by industry and successfully applied to some practical projects $[14,15,17,18]$. Further investigations on VSDs of belt conveyors are carried out in [13]. The current implementations of speed control however concentrate mostly on lower control loops or an individual belt conveyor $[15,16]$. It has not been used to deal with the system constraints and the external constraints, such as time-of-use (TOU) tariff and storage capacities, nor has it been applied to coordinate multiple components of a conveying system.

We intend to use the methodology of optimization to improve the operation efficiency of belt conveyors. Our approach will be based on an energy model of belt conveyors. There exist several 
energy calculation models for the drive system design of belt conveyors [19-23]. These models originate from well-known standards or specifications, such as ISO 5048, DIN 22101, JIS B 8805, and Conveyor Equipment Manufacturers Association (CEMA). They employ either complicated equations or inaccurate empirical constants for the calculation of the energy consumption of belt conveyors. These models are suitable for the design purpose and can hardly be used for optimization calculation. In [24], a new model, characterized by two compensation length variables, is proposed along with a comparative study of the existing energy models. Existing energy models are mostly built under the design conditions. When a belt conveyor operates away from its design condition, inevitably, these models will result in large differences of energy calculation. In practice, most belt conveyors are not working under the design conditions and some of them are working far away from their design conditions, for instance, some belt conveyors even operate with empty belts. Dynamic models of belt conveyors are also studied in the literature. In [26], a dynamic model of belt conveyors is proposed for belt simulation and monitoring. Another dynamic model, based on the spring-mass model, is built in [25]. A third dynamic model of belt conveyors with multiple drives is investigated in [27]. These dynamic models focus mainly on the analysis of transient behavior of belt conveyors, consequently, they are typically not used for energy optimization.

On the other hand, an energy model suitable for optimization purpose will require that it is possible to estimate its parameters through field experiments instead of through design parameters only.

The main purposes of this paper are to build an energy model suitable for optimization and to put optimization to belt conveyors for optimal operation efficiency. We begin with the analysis of the existing energy models. Then an analytical energy model will be proposed. It lumps all the parameters into four coefficients which can be derived from the design parameters or be identified through the technique of parameter identification. Least square (LSQ) [28-31] and recursive least square (RLSQ) [32] are two widely used techniques of parameter estimate in industrial sector $[30,32,33]$. Consequently, we will choose LSQ and RLSQ for off-line and on-line estimation of the four coefficients of the new energy model, respectively. After getting the energy model, we introduce optimization to belt conveyors for optimal energy efficiency. Specifically, the optimization will be done at the operational level with two performance indicators, energy cost and energy consumption, employed as the objectives of optimization. In addition, the time-of-use (TOU) tariff, the feed rate constraint, and belt the speed constraint will be taken into account as well. A typical configuration of belt conveyor system will be used as a case study, where six optimization problems under six different operational conditions will be investigated. The simulation results will be presented.

The layout of the paper is as follows: In Section 2, the existing energy models are reviewed; and an analytical energy model is proposed. Section 3 proposes the off-line and on-line parameter estimation of the new energy model. In Section 4, the optimization of the operation efficiency of belt conveyors is investigated with a case study. The last section is conclusion.

\section{Energy models}

A typical belt conveyor is shown in Fig. 1. The existing energy calculation models of belt conveyors can be divided into two categories. ISO 5048 [19], DIN 22101 [20], and CEMA are based on the methodology of resistance calculation; while JIS B 8805 [21] and Goodyear's model [23] are based on the methodology of energy conversion.

\subsection{Resistance based energy models}

The first category of energy model is characterized by getting energy consumption through the calculation of the resistances. Under the stationary operating condition, the energy consumption of belt conveyors is mainly determined by the resistances to motion of the belt conveyor. ISO 5048 and DIN 22101 divide the motion resistances into four groups: the main resistance, $F_{H}$, the secondary resistance, $F_{N}$, the slop resistance, $F_{s t}$, and the special resistance, $F_{S}$. The peripheral driving force, $F_{U}$, required on the driving pulley(s) of a belt conveyor is obtained by adding up the four groups as follows $F_{U}=F_{H}+F_{N}+F_{S}+F_{s t}$.

When $F_{U}$ is obtained, the mechanical power of a belt conveyor is obtained by

$P_{T}=F_{U} V$

where $V$ is the belt speed in meters per second. Then the power of the motor is $P_{M}=P_{T} / \eta$, where $\eta$ is the overall efficiency of the driving system. Now, the energy calculation is cast to the calculation of the four groups of resistances.

In ISO 5048 and DIN 22101, the main resistance is calculated by

$F_{H}=f L g\left[Q_{R 0}+Q_{R U}+\left(2 Q_{B}+Q_{G}\right) \cos \delta\right]$,

where $f$ is the artificial friction factor, $L$ is the center-to-center distance $(\mathrm{m}), Q_{R 0}$ is the unit mass of the rotating parts of carrying idler rollers $(\mathrm{kg} / \mathrm{m})$, as shown in Fig. $1, Q_{R U}$ is the unit mass of rotating parts of the return idler rollers $(\mathrm{kg} / \mathrm{m}), Q_{B}$ is the unit mass of the belt $(\mathrm{kg} / \mathrm{m}), \delta$ is the inclination angle $\left(^{\circ}\right)$, and $Q_{G}$ is the unit mass of the load $(\mathrm{kg} / \mathrm{m})$. Further, $Q_{G}$ is determined by $Q_{G}=\frac{T}{3.6 \mathrm{~V}}$, where $T$ is the feed rate of the belt conveyor (t/h). Eq. (3) is a simplified form of the main resistance suitable for engineering application, while [34] proposes a non-linear model of the main resistance, which is more complicated. The secondary resistance, in ISO 5048 and DIN 22101, is further divided into four parts: the inertia and frictional resistance at the loading point and in the acceleration area between the material and the belt, $F_{b A}$, the frictional resistance between the skirt boards and the material in the accelerating area, $F_{f}$, the wrap resistance between the belt and the pulley, $F_{w}$, and the pulley bearing resistance, $F_{t}$. Similarly, the special resistance is further divided into four parts: the resistance due to idler tilting, $F_{f r}$, the resistance due to friction between the material handled and the skirt boards, $F_{s b}$, the frictional resistance due to the belt cleaners, $F_{c}$, and the resistance from the material ploughs, $F_{p}$. Accordingly, ISO 5048 and DIN 22101 provide the detailed equations for each part of the secondary resistance and the special resistance. The slop resistance is resulted from the elevation of the material on inclined conveyors. It can be accurately calculated by $F_{s t}=Q_{G} H g$, where $H$ is the net change in elevation ( $\mathrm{m})$.

\subsection{Energy conversion based models}

In view of energy conversion, the power of belt conveyors, under stationary condition, can be divided into three parts as follows [21,23]:

1. the power to run the empty conveyor, $P_{e c}$;

2. the power to move the material horizontally over a certain distance, $P_{h}$;

3. the power to lift the material a certain height, $P_{l}$.

The accessories also contribute to the total power of the belt conveyor, which is denoted by $P_{\text {Acs }}$. The power of the a belt conveyor can be expressed as follows

$P_{T}=P_{e c}+P_{h}+P_{l}+P_{\text {Acs }}$. 


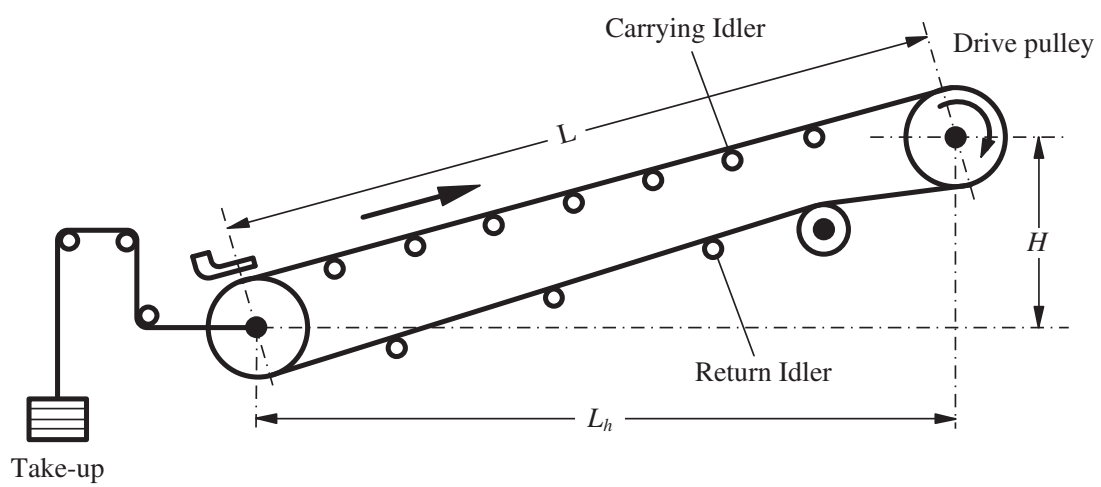

Fig. 1. Typical profile of belt conveyors.

$P_{e c}, P_{h}$ and $P_{l}$ are further calculated by the following empirical formulae, respectively

$P_{e c}=g f\left(L_{h}+L_{0}\right) Q V$,

$P_{h}=g f\left(L_{h}+L_{0}\right) \frac{T}{3.6}$,

$P_{l}=g H \frac{T}{3.6}$,

where $L_{h}$ is the horizontal center-to-center distance $(\mathrm{m}), L_{0}$ is a compensation length constant $(\mathrm{m})$, and $Q=Q_{R 0}+Q_{R U}+2 Q_{B}$. Most frictional resistance components vary directly with the length of belt. However, there exist a few components which are independent of the belt length. $L_{0}$ is consequently used to compensate these components. JIS B 8805 [21] and Goodyear's model are all based on this methodology, however, the determination of the $L_{0}$ 's of these models is quite different [24].

\subsection{Remarks on the existing models}

The resistance based models consider almost all the issues contributing to the energy consumption, thus they are believed to be more accurate. Correspondingly, complicated equations, along with many detailed parameters, are needed for the calculation of these issues. It leads to complexity of calculation. On the other hand, the energy conversion based models simplify the calculation by integrating the compensation length constant(s) into the models. Because these models use one or a few constants to compensate all the cases, their accuracy is usually compromised. Furthermore, all these existing models use the design parameters to calculate the power of belt conveyors. When they are applied to practical condition, large difference of energy calculation will be generated because the practical operation condition always deviates from the design one.

\subsection{An analytical energy model}

An analytical energy model is proposed here to meet the requirement of energy optimization. It has its root in ISO 5048 [19], however, its analytical form makes it suitable for parameter estimation and energy optimization.

According to ISO 5048 and DIN 22101, the secondary resistance of a belt conveyor is obtained from four parts as follows

$F_{N}=F_{b A}+F_{f}+F_{w}+F_{t}$.

$F_{t}$ is relatively small, hence it can be omitted [20]. $F_{w}$ is also small and does not vary much, so it is taken as a constant, $C_{F t}$. If the initial speed of material in the direction of belt movement is taken as zero and the frictional factor between the material and the belt is taken as the same as that between the material and the skirt boards [19],
$F_{b A}$ and $F_{f}$ can be obtained through the following two equations, respectively

$F_{b A}=\frac{T V}{3.6}$

$F_{f}=\frac{T^{2}}{6.48 \rho b_{1}^{2}}$,

where $\rho$ is the bulk density of material $\left(\mathrm{kg} / \mathrm{m}^{3}\right)$, and $b_{1}$ is the width between the skirt boards $(\mathrm{m})$. Now, $F_{N}$ can be rewritten as follows

$F_{N}=\frac{T V}{3.6}+\frac{T^{2}}{6.48 \rho b_{1}^{2}}+C_{F t}$.

The special resistances, $F_{S}$, for an existing belt conveyor, including $F_{f r}, F_{s b}, F_{c}$ and $F_{p}$, has the following relation with $T$ and $V$ [19]

$F_{S}=k_{1} \frac{T^{2}}{V^{2}}+k_{2} \frac{T}{V}+k_{3}$

where $k_{1}, k_{2}$, and $k_{3}$ are constant coefficients which relate to the structural parameters of the belt conveyor.

Combining (3), (8) and (9), and $F_{s t}=Q_{G} H g$ with (2), we get

$$
\begin{aligned}
P_{T}= & \frac{V^{2} T}{3.6}+\frac{V T^{2}}{6.48 \rho b_{1}^{2}} \\
& +\left\{g f Q\left[L \cos \delta+L(1-\cos \delta)\left(1-\frac{2 Q_{B}}{Q}\right)\right]+k_{3}+C_{F t}\right\} V \\
& +k_{1} \frac{T^{2}}{V}+\left(\frac{g L \sin \delta+g f L \cos \delta}{3.6}+k_{2}\right) T .
\end{aligned}
$$

Further, let

$$
\begin{aligned}
\theta_{1} & =\frac{1}{6.48 b_{1}^{2} \rho}, \\
\theta_{2} & =g f Q\left[L \cos \delta+L(1-\cos \delta)\left(1-\frac{2 Q_{B}}{Q}\right)\right]+k_{3}+C_{F t}, \\
\theta_{3} & =k_{1}, \\
\theta_{4} & =\frac{g L \sin \delta+g f L \cos \delta}{3.6}+k_{2},
\end{aligned}
$$

we get the analytical energy model of a belt conveyor as follows

$P_{T}-\frac{V^{2} T}{3.6}=\theta_{1} T^{2} V+\theta_{2} V+\theta_{3} \frac{T^{2}}{V}+\theta_{4} T$.

$\theta_{1}, \theta_{2}, \theta_{3}$ and $\theta_{4}$ are determined by the structural parameters and components of a belt conveyor, by the operation circumstance and by the characteristic of the material handled, therefore, they are relatively constant for a certain belt conveyor. Because (12) originates from ISO 5048, it can be used for design purpose as well, where $\theta_{1}, \theta_{2}, \theta_{3}$, and $\theta_{4}$ are derived from design parameters. In 
practice, maintenance, readjustment, retrofit, abrasion and circumstance change probably make a belt conveyor away from its design condition, consequently, changes of the parameters $\theta_{1}, \theta_{2}, \theta_{3}$, and $\theta_{4}$. Hence, when (12) is applied to a practical belt conveyor for energy optimization, its four coefficients should be estimated through experiments instead of be derived from design parameters.

\section{Parameter estimate}

In (12), if $P_{T}, V$, and $T$ are measured on-line or off-line, $\theta_{1}, \theta_{2}, \theta_{3}$ and $\theta_{4}$ can be estimated from these data to guarantee the accuracy of energy model. Actually, $P_{T}$ is the mechanical power of a belt conveyor, it can hardly be measured directly, however, it can be indirectly obtained from the electric power of the motor by $P_{T}=\eta P_{M}$. In practice, power meters, encoders and electronic belt scales are usually equipped with belt conveyors to obtain $P_{M}, V$ and $T$, respectively. For belt conveyors without permanent instruments for $P_{M}, V$ and $T$, the off-line parameter estimation is employed, where temporary instruments, usually portable ones, will be used for necessary experiments. On the other hand, if the belt conveyors are equipped with permanent instruments, the on-line estimation will be carried out.

\subsection{Off-line parameter estimation with LSQ}

For the principle of LSQ, please refer [28,29]. Because the analytical energy model is linear, LSQ will yield satisfactory results when applied to this model. It is reasonable to normalize the variables before parameter estimation. The analytical model (12) can be rewritten as

$$
\begin{aligned}
\frac{P_{T}}{M}-\frac{V^{2} T}{3.6 M}= & \left(\frac{m_{1}}{M} \theta_{1}\right) \frac{T^{2} V}{m_{1}}+\left(\frac{m_{2}}{M} \theta_{2}\right) \frac{V}{m_{2}}+\left(\frac{m_{3}}{M} \theta_{3}\right) \frac{T^{2}}{m_{3} V} \\
& +\left(\frac{m_{4}}{M} \theta_{4}\right) \frac{T}{m_{4}},
\end{aligned}
$$

where $m_{1}=\max \left(T^{2} V\right), m_{2}=\max (V), m_{3}=\max \left(T^{2} / V\right), m_{4}=\max (T)$, and $M=\max \left(P_{T}-V^{2} T / 3.6\right)$. Denoting

$$
\begin{aligned}
& \Psi=\left[\begin{array}{llll}
\frac{m_{1}}{M} \theta_{1} & \frac{m_{2}}{M} \theta_{2} & \frac{m_{3}}{M} \theta_{3} & \frac{m_{4}}{M} \theta_{4}
\end{array}\right]^{T}, \\
& \Delta=\left[\begin{array}{llll}
\frac{T^{2} V}{m_{1}} & \frac{V}{m_{2}} & \frac{T^{2}}{m_{3} V} & \frac{T}{m_{4}}
\end{array}\right]^{T},
\end{aligned}
$$

and

$P_{T N}=\frac{P_{T}}{M}-\frac{V^{2} T}{3.6 M}$,

we transform (13) as follows

$P_{T N}=\Psi^{T} \cdot \Delta$.

During the field experiments, the electric power, belt speed, and feed rate are recorded at different time. $P_{M}(k), V(k)$ and $T(k)$ denote the readings of the instruments of the $k$ th experiment, where $k$ is the number of the experiment, $1 \leqslant k \leqslant N$ and $N$ is the total number of experiments. Then, $P_{T}(k)$ is generated from $P_{M}(k)$ by $P_{T}(k)=\eta P_{M}(k)$. At least four experiments are needed to estimate the four coefficients of (12), in other words, $N \geqslant 4$. Denoting

$H=\left[\begin{array}{c}\Delta^{T}(1) \\ \vdots \\ \Delta^{T}(N)\end{array}\right]$,

and

$Z=\left[\begin{array}{lll}P_{T N}(1) & \ldots & P_{T N}(N)\end{array}\right]^{T}$,

we get the least square estimation of $\Psi$ as follows [29]

$$
\widehat{\Psi}=\left(H^{T} H\right)^{-1} H^{T} Z .
$$

If $H^{T} H$ is invertible, there is an unique solution to $\Psi$, accordingly, the estimation of $\Theta=\left[\begin{array}{llll}\theta_{1} & \theta_{2} & \theta_{3} & \theta_{4}\end{array}\right]^{T}$ can be obtained by

$$
\widehat{\Theta}=\widehat{\Psi} \cdot\left[\begin{array}{llll}
\frac{M}{m_{1}} & \frac{M}{m_{2}} & \frac{M}{m_{3}} & \frac{M}{m_{4}}
\end{array}\right]^{T} .
$$

In case of constant $V$ or $T, H^{T} H$ becomes singular, this LSQ based parameter estimation fails. Hence, the field experiments should be carefully designed to avoid these cases. The recommended procedure for off-line parameter estimation is shown as follows.

1. Install instruments for $P_{M}, V$ and $T$. If the belt conveyor has permanent instruments already, this step is unnecessary.

2. Control $V$ or $T$ to operate the belt conveyor at different operating points and record the readings of $P_{M}, T$ and $V$. At least four experiments are needed. Running with empty belt, $T=0$, is considered as a specific operating point of a belt conveyor. It is not mandatory for the experiment, however, if permitted, this specific operating point is recommended because it can obtain $\theta_{2}$ directly by a single experiment.

3. All the data are used by LSQ to estimate $\theta_{1}, \theta_{2}, \theta_{3}$ and $\theta_{4}$.

4. The off-line parameter estimation should be carried out periodically, for example once a week, to guarantee the accuracy of the energy model.

\subsection{On-line parameter estimation with RLSQ}

For belt conveyors with permanent instruments for $P_{M}, T$ and $V$, the real-time data can be accessed periodically through the supervisory control and data acquisition system (SCADA) or through the communication interface of the instruments. Under this condition, an on-line parameter estimation can be adopted to adjust the coefficients automatically, consequently, guarantees the accuracy of the energy model. In this paper, RLSQ is used for this purpose.

When the real-time values of $T, V$ and $P_{T}$, derived from $P_{M}$, are obtained, they are also normalized using (13) before feeding to the recursive calculation. However, under on-line parameter estimation, $M, m_{1}, m_{2}, m_{3}$ and $m_{4}$ are not determined by the maximum values of the corresponding combinations. Instead, they are determined by the upper bounds of $\left(P_{T}-V^{2} T / 3.6\right),\left(T^{2} V\right), V,\left(T^{2} / V\right)$ and $T$, respectively. For a certain belt conveyor, the upper bounds of these combinations can be easily estimated.

Denoting

$$
h(i)=\widehat{\Psi} \cdot\left[\begin{array}{llll}
\frac{T(i)^{2} V(i)}{m_{1}} & \frac{V(i)}{m_{2}} & \frac{T(i)^{2}}{m_{3} V(i)} & \frac{T(i)}{m_{4}}
\end{array}\right]
$$

and

$z(i)=\frac{P_{T}(i)}{M}-\frac{V(i)^{2} T(i)}{3.6 M}$

we can obtain the estimation of $\Psi$ using the following recursive formulas [29]:

$$
\begin{aligned}
& \widehat{\Psi}(i)=\widehat{\Psi}(i-1)+K(i)\left[z(i)-h^{T}(i) \widehat{\Psi}(i-1)\right], \\
& P(i)=\left[I-K(i) h^{T}(i)\right] P(i-1), \\
& K(i)=P(i-1) h(i)\left[h^{T}(i) P(i-1) h(i)+\alpha\right]^{-1},
\end{aligned}
$$

where $i$ and $(i-1)$ denote the current data pair and the last data pair, respectively. $\alpha$ in (24) is a weight which may be selected in the interval $0<\alpha \leqslant 1$. With $\alpha=1$, all sampled data pairs are equally weighted. After $\widehat{\Psi}(i)$ is obtained, $\widehat{\Theta}(i)$ is calculated by

$$
\widehat{\Theta}(i)=\widehat{\Psi}(i) \cdot\left[\begin{array}{llll}
\frac{M}{m_{1}} & \frac{M}{m_{2}} & \frac{M}{m_{3}} & \frac{M}{m_{4}}
\end{array}\right]^{T} \text {. }
$$




\subsection{Results of parameter estimation}

Before the on-line and off-line parameter estimation techniques are put to actual belt conveyors, their efficacy with generated data is checked. The belt conveyor in [24] is used for illustration. Firstly, the detailed parameters from [24] are applied to the analytical energy model to get the coefficients as $\Theta=\left[2.3733 \times 10^{-4}\right.$ $\left.8.5663 \times 10^{3} 0.003151 .6804\right]^{T}$. Secondly, suppose $V$ vary from 0 to $3.13 \mathrm{~m} / \mathrm{s}, T$ vary from 0 to $2000 \mathrm{t} / \mathrm{h}$ and $t=0,1,2, \ldots, 100 \mathrm{~min}$, the data pairs of $V, T$, and $P_{T}$ are generated. They simulate the readings of the instruments equipped with the belt conveyor. Thirdly, $1 \%, 3 \%, 5 \%$ and $10 \%$ random components are added to the data pairs to simulate the measurement noise. Now, the generated data pairs are supposed to be close to the real readings of field experiments. It is reasonable to use these data pairs for parameter estimation and verification.

Off-line parameter estimation of the energy model is then investigated with the generated data. The needed data pairs are taken from the generated data as experimental readings and then used for off-line parameter estimation. When applied to the data pairs without random components, the LSQ based parameter estimation achieves near-perfect estimation of coefficients. The data pairs with approximately the same belt speeds or approximately the same feed rates are used for investigation as well. These specific data pairs make $H^{T} H$ singular or near-singular, consequently, they result in failure or large errors of parameter estimation. Subsequently, various amounts of data pairs, ranges from 4 to 10 , along with different noise $(1 \%, 3 \%, 5 \%$ and $10 \%)$ are investigated. Specifically, we use the cases with four and ten data pairs for demonstration. The results are shown in Fig. 2. It is found that the offline parameter estimation with four data pairs fails with noisy measurements, on the contrary, the estimation with ten data pairs shows good robustness against measurement noise. Hence, if permitted by the field conditions, more experiments should be carried out to guarantee the accuracy of estimation. The influence from measurement noise is also demonstrated in Fig. 2. The data pairs with small noise yield relatively accurate estimation. Therefore, the accurate measurement instruments are recommended for field experiments.

Subsequently, the generated data are applied to on-line parameter estimation. Using the data without random components, the on-line parameter estimation achieves near-perfect estimation of the coefficients. Theoretically, four categories of data may be obtained from an individual belt conveyor: data with variable $V$ and variable $T$, data with variable $V$ and constant $T$, data with constant $V$ and variable $T$ and those with constant $V$ and constant $T$.

The generated data with variable $V$ and variable $T$, subject to various measurement noise ( $1 \%, 3 \%$ and $5 \%$ ), are firstly applied to the on-line parameter estimation. The result is shown as Fig. 3. It is found that the measurement noise affects both the convergence speed and the accuracy of coefficient estimates. In particular, the accuracy of $\theta_{2}$ is greatly affected by the measurement noise. Subsequently, the other three categories of data, subject to the same measurement noise (3\%), are put to the on-line parameter estimation to get the result as shown in Fig. 4. The category with constant $V$ and constant $T$ is unpractical, hence, it is excluded from the investigation. It is found from Fig. 4 that this RLSQ based on-line parameter estimate works well with the data with variable $V$ and variable $T$. When subject to the data, where one of the two variables, $V$ and $T$, keeps constant, this on-line parameter estimation works as well, but $\theta_{1}$ and $\theta_{2}$ converge to different values with acceptable difference from the exact ones. The convergence deviation results from the constancy of $V$ or $T$, which decreases the information of the data collected for parameter estimation. Practically, the belt conveyors usually work with variable $V$ and variable $T$, the on-line parameter estimation works well under this condition. It guarantees the practicability of the on-line parameter estimation of the energy model.

\section{Energy efficiency optimization}

Energy efficiency optimization of belt conveyors, making financial and environmental sense, is the ultimate purpose of this paper. The newly proposed analytical energy model makes the energy optimization of belt conveyors feasible. Further, the off-line and on-line estimations make the energy model relatively accurate, consequently, make the optimization practical. Operation efficiency of an energy system is improved through the coordination of two or more internal sub-systems, or through the coordination of the system components and time, or through the coordination of the system and human operators [3]. In the case of a belt conveyor system, its operation efficiency can be improved through the coordination of its belt speed and feed rate or through the coordination of its operational status and time. Actually, the coordination of belt speed and feed rate is reflected as the variable speed drive or speed control, on the other hand, the coordination of its operational status and time is reflected as load shifting. This

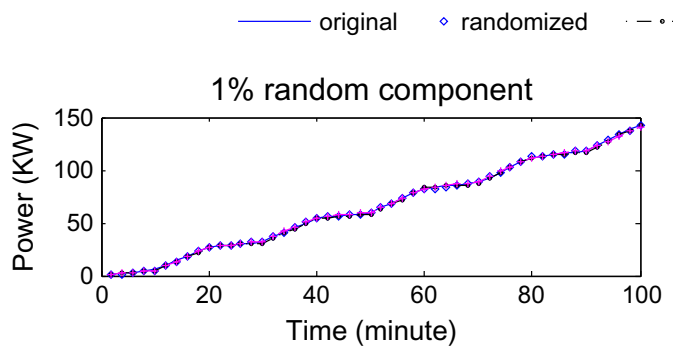

-4 experiments -+-10 experiments
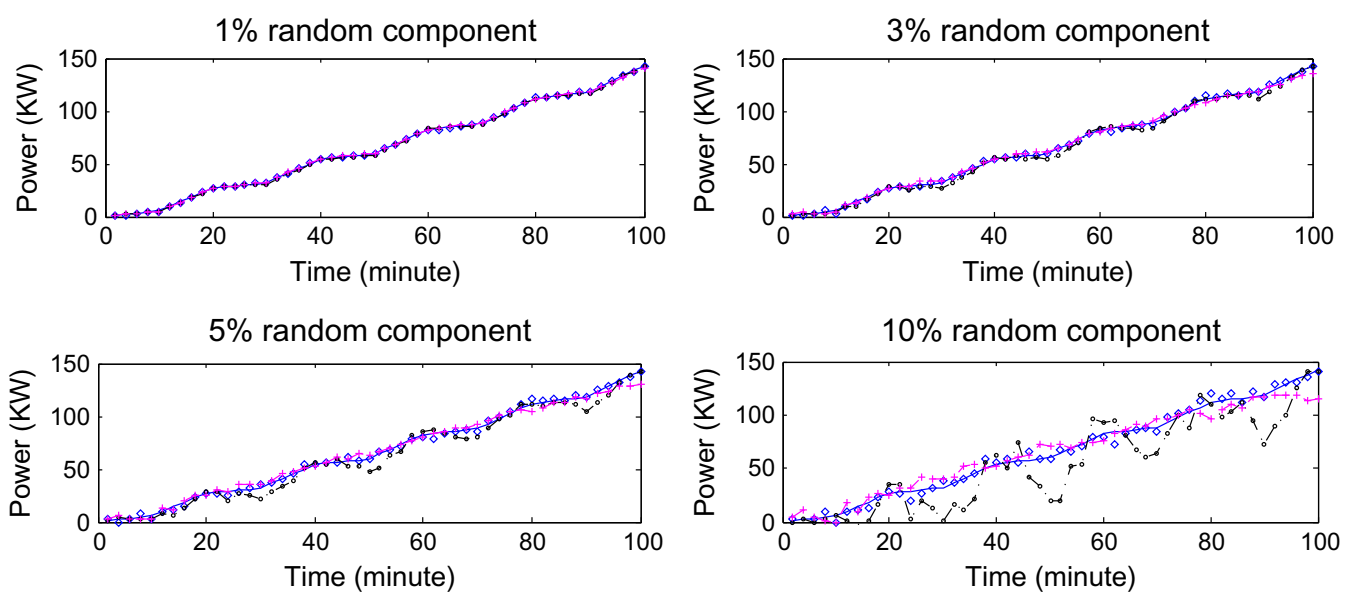

Fig. 2. Off-line parameter estimation on randomized data. 

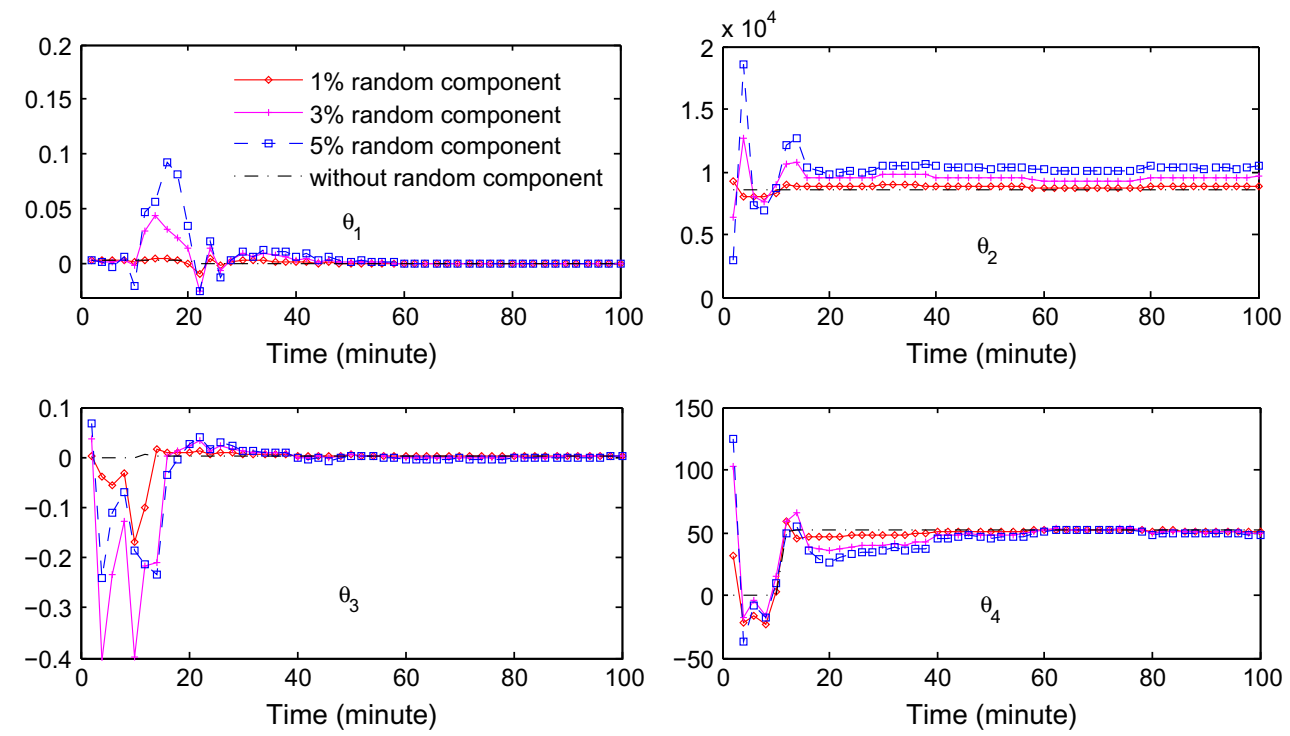

Fig. 3. On-line parameter estimation with randomized data.
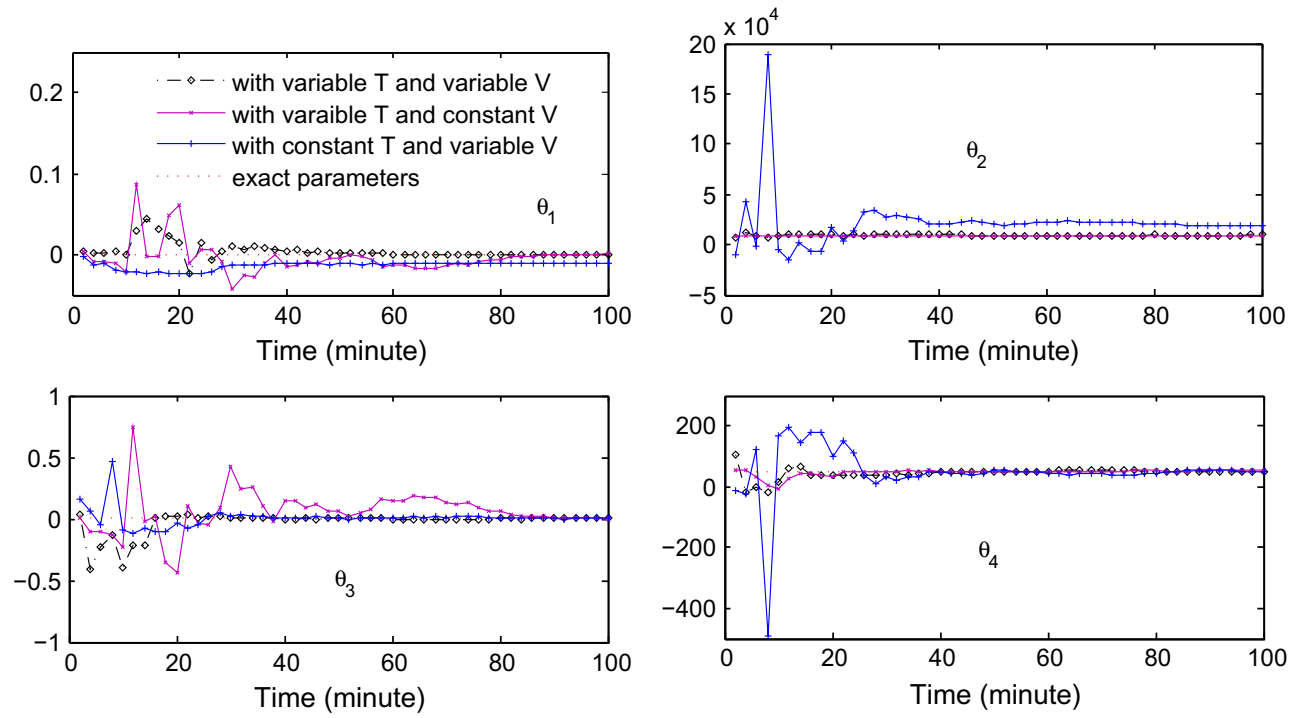

Fig. 4. On-line parameter estimation with various data categories.

section begins with an analysis on the newly proposed energy model, and then introduces optimization to improve the operation efficiency of belt conveyors with the consideration of various constraints.

\subsection{Analysis of the energy model}

A 3D plot of the analytical energy model is shown in Fig. 5 for better understanding of the operation efficiency improvement through speed control. Practically, the belt conveyor operates between $V=V_{\text {MAX }}$ plane and $T=3.6 Q_{G_{-M A X}} V$ plane as shown in Fig. 5, where $V_{\text {MAX }}$ is the upper bound of belt speed and $Q_{G_{-} \text {MAX }}$ is the upper bound of unit mass of load which is determined by the characteristics of the belt and the material being transported. Under certain feed rate, e.g., $T=1000 \mathrm{t} / \mathrm{h}$ as shown in Fig. 5, $A, B$ and $C$ are the possible operating points of the belt conveyor. Point $A$ is on $V=V_{\text {MAX }}$ plane, point $C$ is on $T=3.6 Q_{G_{-M A X}} V$ plane, while $B$ is on any plane between the two planes. It is clearly shown in Fig. 5 that

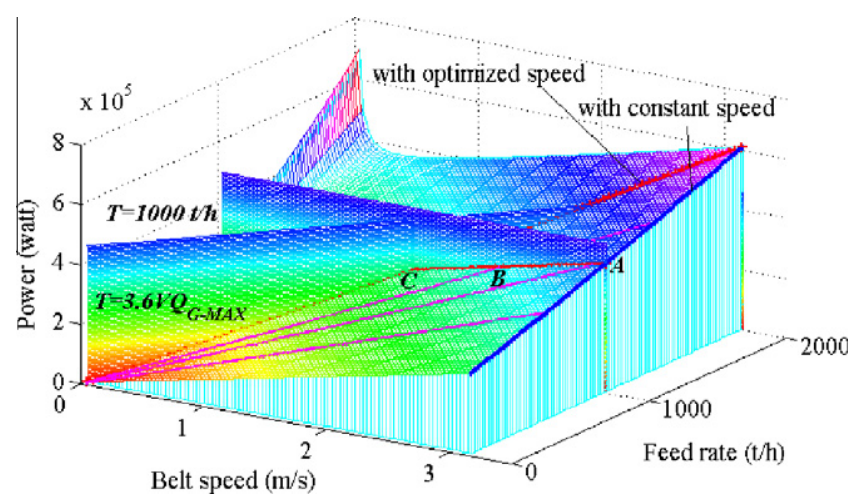

Fig. 5. 3D plot of the analytical energy model.

while working with the same production, reflected by $T$, operating point $C$ consumes less power than $B$ and $A$. In other words, energy 
saving is achieved through speed control which reduces the belt speed to keep a high amount of material on the belt. This is also the aim of the current lower control loops.

\subsection{Operation efficiency optimization}

Belt conveyors always work along with other equipment, e.g., feeders and stockpiles, to meet certain task of material handling. Consequently, the operation of belt conveyors should concentrate on not only energy efficiency but also certain constraints at equipment level or system level. The current implementation of speed control on belt conveyors employs lower control loops to improve energy efficiency, however it can hardly deal with various constraints, especially, the ones at system level. We intend to put optimization to belt conveyors to obtain optimal operation efficiency and deal with various constraints at the same time. We take a belt conveyor, conveying bulk material from one location to another, as shown in Fig. 6, for optimization. This is a typical configuration of belt conveyor system, hence, it can be used for a general demonstration.

The following assumptions are made in order to model the belt conveyor system in Fig. 6 as simplified optimization problems.

1. At any time, silo $A$ has enough material to supply the belt conveyor; and stockpile $B$ always has enough capacity to store the material.

2. The time delay associated with the material from silo $A$ to stockpile $B$ is ignored.

3. The dynamic energy consumption associated with start-up and stop of the belt conveyor is not taken into account.

Energy model (12) calculates the mechanical power of a belt conveyor. Incorporated with the efficiency of the drive system, model (12) is rewritten as follows

$f_{P}(V, T)=\frac{1}{\eta}\left(\theta_{1} V T^{2}+\theta_{2} V+\theta_{3} \frac{T^{2}}{V}+\theta_{4} T+\frac{V^{2} T}{3.6}\right)$,

where $f_{P}(V, T)$ is the electrical power of the drive motor when the belt conveyor is working with the belt speed $V$ and the feed rate $T$. The efficiency of the entire drive system is obtained by $\eta=\eta_{d} \cdot \eta_{m}$, where $\eta_{m}$ is the efficiency of motor and $\eta_{d}$ is the efficiency of the drive. Using the energy model (26), several optimization problems for belt conveyors will be formulated. To consider optimal operation efficiency of belt conveyers, we take energy consumption or energy cost, the typical indicators to measure performance efficiency, as the objectives of the following optimization problems instead of a direct indicator of operation efficiency, because the performance efficiency can drive the operation in its optimal efficiency and possibly balance the performance indicator cost and a technical specification.

For a belt conveyor, the total energy consumption, $J_{E}$, is related to the electrical power and the time period for calculation. It can be expressed as an integration of the electrical power, $f_{P}(V, T)$, between $t_{0}$ and $t_{f}$ as follows

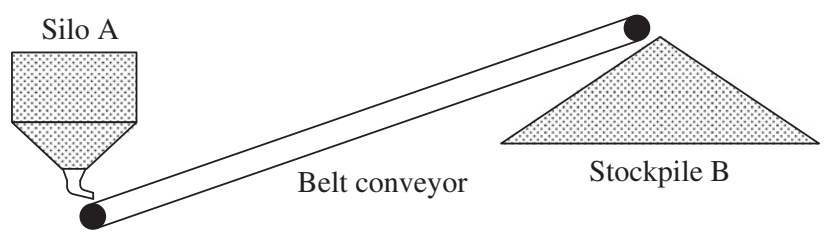

Fig. 6. Belt conveyor system for optimization.
$J_{E}=\int_{t_{0}}^{t_{f}} f_{P}(V(t), T(t)) d t$

where $\left[t_{0} t_{f}\right]$ is the time period for total energy consumption calculation. Similarly, the total energy cost, $J_{C}$, can be calculated as follows

$J_{C}=\int_{t_{0}}^{t_{f}} f_{P}(V(t), T(t)) p(t) d t$

where $p(t)$ is the TOU tariff function. For ease of discrete-time numerical analysis, the energy consumption function (27) and the cost function (28) are discretized. Let the sampling time $t_{s}=\frac{t_{f}-t_{0}}{N}$, we can obtain the discrete form of the total energy consumption and total energy cost as

$J_{E}=\sum_{j=1}^{N} f_{P}\left(V_{j}, T_{j}\right) t_{s}$

and

$J_{C}=\sum_{j=1}^{N} f_{P}\left(V_{j}, T_{j}\right) p_{j} t_{s}$,

respectively, where $V_{j}, T_{j}$ and $p_{j}$ are the belt speed, the feed rate, and the electricity price at the $j$ th sample time. $J_{E}$ and $J_{C}$ are performance indicators, which are to be employed as the objectives of the following optimization problems except the first one.

\subsubsection{Optimization problem one}

In practice, many belt conveyors are working with reduced feed rates, even with empty belts, due to mismatched feeders, material blockage, or improper operation. Optimization problem one is dedicated to optimize the belt conveyors under a reduced feed rate, $T_{R E D}$. The basic aim is to match the belt speed with the given reduced feed rate to minimize the energy consumption. Hence, electrical power is employed as the objective of this problem for minimization. The belt speed should be within its feasible domain, $0 \leqslant V \leqslant V_{M A X}$; and the unit mass should be within its feasible domain, $0 \leqslant Q_{G} \leqslant Q_{G_{-M A X}}$, as well. They form the constraints of this problem. Hence, optimization problem one is formulated as follows

$\min J_{P}(V)=f_{P}\left(V, T_{R E D}\right)$,

subject to $T=T_{R E D}$,

$$
\begin{aligned}
& 0 \leqslant V \leqslant V_{\text {MAX }}, \\
& 0 \leqslant Q_{G} \leqslant Q_{G_{-} \text {MAX }} .
\end{aligned}
$$

The solution to this problem, $\bar{V}$, is the optimal belt speed according to the given reduced feed rate, $T_{R E D}$.

\subsubsection{Optimization problem two}

For a belt conveyor, as shown in Fig. 6, generally, there is a total production requirement, $T_{S U M}$, over a certain time period, $\left[t_{0} t_{f}\right]$. In this optimization problem, the total energy consumption is taken as the objective for minimization. Three variables, belt speed, feed rate and working time, denoted by $t_{w}$, will be optimized to minimize the total energy consumption. The constraints for this optimization problem are listed as follows. (i) The belt speed should be within its feasible domain, $0 \leqslant V \leqslant V_{\text {MAX. }}$ (ii) The unit mass should be within its feasible domain, $0 \leqslant Q_{G} \leqslant Q_{G_{-} \text {MAX. }}$. (iii) The total production of the belt conveyor is great than or equal to its requirement, $T \cdot t_{w} \geqslant T_{\text {SUM }}$. (iv) The working time is within $\left[\begin{array}{ll}t_{0} & t_{f}\end{array}\right]$, $0 \leqslant t_{w} \leqslant\left(t_{f}-t_{0}\right)$. Eventually, optimization problem two is formulated as 
$\min J_{P}\left(V, T, t_{w}\right)=f_{P}(V, T) t_{w}$,

subject to $0 \leqslant V \leqslant V_{\text {MAX, }}$

$0 \leqslant Q_{G} \leqslant Q_{G_{-} M A X}$

$T \cdot t_{w} \geqslant T_{\text {SUM }}$

$0 \leqslant t_{w} \leqslant\left(t_{f}-t_{0}\right)$.

$V, T$, and $t_{w}$ are the optimization variables of this problem. The solution, denoted by $\bar{V}, \bar{T}$, and $\bar{t}_{w}$, is the optimal operational instruction, which schedules the belt speed, feed rate, and working time of the belt conveyor optimally to minimize the energy consumption subject to the above constraints.

\subsubsection{Optimization problem three}

This optimization problem considers the energy cost of the belt conveyor subject to TOU tariff. Generally, the operation efficiency of an energy system contributes to its performance efficiency, reversely, performance efficiency can drive the operation in its optimal efficiency. Hence, it is reasonable to take $J_{C}$ in (30), a performance indicator, as the objective of this problem. In this problem, the optimization variables, $V$ and $T$, are vectors instead of scalar as in problem one and two. Optimization problem three shares the first two constraints of problem two. However, the third one, concerning the total production, is lightly different from that of problem two and should be expressed as

$\sum_{j=1}^{N} T_{j} t_{s} \geqslant T_{\text {SUM }}$

Hence, this optimization problem is finally formulated as follows

$$
\min J_{C}\left(V_{j}, T_{j}: 1 \leqslant j \leqslant N\right)=\sum_{j=1}^{N} f_{P}\left(V_{j}, T_{j}\right) p_{j} t_{s},
$$

subject to $0 \leqslant V_{j} \leqslant V_{\text {MAX }}$,

$$
0 \leqslant Q_{G_{-} j} \leqslant Q_{G_{-} M A X}
$$$$
\sum_{j=1}^{N} T_{j} t_{s} \geqslant T_{\text {SUM }}
$$

The solution to this problem, $\left[\bar{V}_{j}, \bar{T}_{j}: 1 \leqslant \mathrm{j} \leqslant \mathrm{N}\right]$, is the operational instructions for the belt conveyor, where $\bar{V}=\left[\bar{V}_{1}, \bar{V}_{2}, \ldots, \bar{V}_{N}\right]$ and $\bar{T}=\left[\bar{T}_{1}, \bar{T}_{2}, \ldots, \bar{T}_{N}\right]$.

\subsubsection{Optimization problem four}

This optimization problem is similar to problem three. The difference between the two is that optimization problem four takes an extra issue, ramp rate of belt speed, into account during the optimization. In practice, large ramp rate of belt speed does harm to certain equipment or components of the belt conveyor. One way to reduce the ramp rate of belt speed is to integrate it into the objective function for minimization. Thus, an additional part, $\sum_{j=1}^{N-1}\left(V_{j+1}-V_{j}\right)^{2}$, is added to the objective function (30). The modified objective function is expressed as follows

$J_{C}=\sum_{j=1}^{N} f_{P}\left(V_{j}, T_{j}\right) p_{j} t_{s}+\varpi \sum_{j=1}^{N-1}\left(V_{j+1}-V_{j}\right)^{2}$,

where $\varpi$ is a weight, which is employed to balance the economic performance and the technical specification. A second way to consider the ramp rate constraints is to directly impose lower and upper bounds for $\left(V_{j+1}-V_{j}\right)$. A third way is to further model the dynamics of the drive systems, so, dynamical constraints of $V_{j+1}$ and $V_{j}$ can be established. In this paper, the first way is employed for simplicity purpose. Another reason for us to build a technical constraint into the objective function is to show that a performance efficiency indicator can represent a balance between an economic indicator and a technical indicator, as will be shown in the following. Thus, optimization problem four is formulated as

$$
\min J_{C}\left(V_{j}, T_{j}: 1 \leqslant j \leqslant N\right)=\sum_{j=1}^{N} f_{P}\left(V_{j}, T_{j}\right) p_{j} t_{s}+\varpi \sum_{j=1}^{N-1}\left(V_{j+1}-V_{j}\right)^{2},
$$

subject to $0 \leqslant V_{j} \leqslant V_{M A X}$,

$$
\begin{aligned}
& 0 \leqslant Q_{G_{-} j} \leqslant Q_{G_{-} M A X}, \\
& \sum_{j=1}^{N} T_{j} t_{s} \geqslant T_{S U M} .
\end{aligned}
$$

\subsubsection{Optimization problem five}

If the material is fed to belt conveyor by tripper cars, trucks or some other feeding devices with intermittent characteristic, the feed rate becomes uncontrollable. Under this condition, the optimization is to match the belt speed with feed rate. Further, if the feed rate is predictable, the optimal schedule of belt conveyor can be carried out. This assumption comes from the fact that the working time of the intermittent feeding devices, e.g., tripper cars or trucks, is uncontrollable but predicable, further, when these feeding devices are working their feed rates are predictable as well. Again, we take the energy consumption as the objective of this optimization problem. The constraints of this problem originate from the belt speed limit and unit mass limit. Then, optimization problem five can be formulated as

$$
\min J_{C}\left(V_{j}: 1 \leqslant j \leqslant N\right)=\sum_{j=1}^{N} f_{P}\left(V_{j}, T_{j}\right) t_{s}
$$

subject to $0 \leqslant V_{j} \leqslant V_{M A X}$,

$$
0 \leqslant Q_{G_{-} j} \leqslant Q_{G_{-} M A X},
$$

where $T=\left[T_{1}, T_{2}, \ldots, T_{N}\right]$ is predicted in advance and $V$ is the optimization vector. The solution to this problem, $\bar{V}=\left[\bar{V}_{1}, \bar{V}_{2}, \ldots, \bar{V}_{N}\right]$, optimally matches belt speeds with the predicted feed rates to obtain optimal operation efficiency.

\subsubsection{Optimization problem six}

This problem is similar to problem five. It adds an additional part $\varpi \sum_{j=1}^{N-1}\left(V_{j+1}-V_{j}\right)^{2}$ to the objective function as problem four does to reduce the ramp rate of belt speed. This is the only difference between problem five and problem six, hence, the formulation of this problem is omitted. In fact, optimization problem four and six balance an economic performance indicator and a technical indicator. The second one originates from the equipment level. In other words, the operation efficiency of belt conveyors can be reflected or driven by indicators of performance level and equipment level.

\subsection{Simulation results}

In this section, the six optimization problems are solved through simulation for the belt conveyor, as shown in Fig. 6. The belt conveyor is supposed to have the same parameters as that used for case study in [24]. Hence, we get the coefficients of energy model as $\Theta=\left[2.3733 \times 10^{-4} 8.5663 \times 10^{3} 0.003151 .6804\right]^{T}$. The efficiency of the motor and the drive, $\eta_{m}$ and $\eta_{d}$, are set to 0.9408 and 0.945 , respectively. The optimization interval and sampling time are set to $24 \mathrm{~h}$ and $10 \mathrm{~min}$, hence, the sample number $N=144$.

The TOU tariff is an important input of problem three and problem four. In this case study, the belt conveyor is supposed to work under the Eskom Megaflex time-of-use (TOU) tariff plan. Consequently, the Eskom Megaflex Active Energy Charge is used. It can be described by 


$$
p(t)= \begin{cases}p_{o}, & \text { if } t \in[0,6) \cup[22,24), \\ p_{s}, & \text { if } t \in[6,7) \cup[10,18) \cup[20,22), \\ p_{p}, & \text { if } t \in[7,10) \cup[18,20),\end{cases}
$$

where $t$ is the time of any day in hours (from 1 to 24); $p_{o}, p_{s}$ and $p_{p}$ are the off-peak, standard and peak TOU energy tariff in $R / \mathrm{kW} \mathrm{h}$, where $R$ is South African currency Rand. The values of $p_{o}, p_{s}$ and $p_{p}$ vary according to the time of day, the day of the week as well as the season. We take the high-demand season [June-August] for investigation, where $p_{o}=0.6304 \mathrm{R} / \mathrm{kW} \mathrm{h}, p_{s}=0.1667 \mathrm{R} / \mathrm{kWh}$ and $p_{p}=0.0906 \mathrm{R} / \mathrm{kW} \mathrm{h}$.

The proposed six optimization problems are real-value optimization problems. All simulations are carried out in the MATLAB environment. The fmincon function of MATLAB Optimization Toolbox is used to solve these problems.

Optimization problem one is firstly investigated. When the reduced feed rate, $T_{R E D}$, ranges from $100 \mathrm{t} / \mathrm{h}$ to $2000 \mathrm{t} / \mathrm{h}$ which is the designed value of the feed rate, this optimization problem is solved repeatedly. The result is shown as Fig. 7. When belt conveyor works under the design condition, the optimized belt speed equals to the constant designed belt speed, $V_{p}$. On the other hand, when reduced feed rate is encountered, the belt conveyor consumes less power with optimized belt speed than that with designed belt speed. It is also clearly shown in Fig. 7 that the further the feed rate is reduced, the more energy can be saved through the optimization of the belt speed.

Optimization problem two, three and four take the total production as a constraint, hence, they are formed as a group for investigation. For the sake of reliability and feasibility, most of the belt conveyors are intentionally over designed, consequently, the belt conveyors fulfill the required tasks with reduced working time. Considering this practical condition, we set the total production requirement, $T_{S U M}$, to $43,200 \mathrm{t}, 33,600 \mathrm{t}$ and $24,000 \mathrm{t}$ for investigation respectively. They equal to $90 \%, 70 \%$ and $50 \%$ of the maximum amount of material that can be transferred by the belt conveyor within $24 \mathrm{~h}$. Similar results are obtained under the three conditions. Specifically, the simulation result with $T_{S U M}=33,600 \mathrm{t}$ is presented as Fig. 8; and the corresponding energy consumption and energy cost are listed in Table 1. Optimization problem four selects two values, 50 and 500, for $\varpi$ to show the influence from the weight.

As shown in Fig. 8, optimization problem two does not consider the TOU tariff and its feed rate and belt speed keep constant all through. For a given total production requirement, the belt speed and feed rate of optimization problem two are reduced in tandem to obtain the minimum energy consumption by extending the working time. The TOU tariff is integrated into the objectives of problem three and problem four. Consequently, the optimal solution to problem three and problem four runs the belt conveyor with maximum capacity during off-peak time, operates the belt conveyor with reduced feed rate and belt speed during standard time, further, stops the belt conveyor during peak time as shown

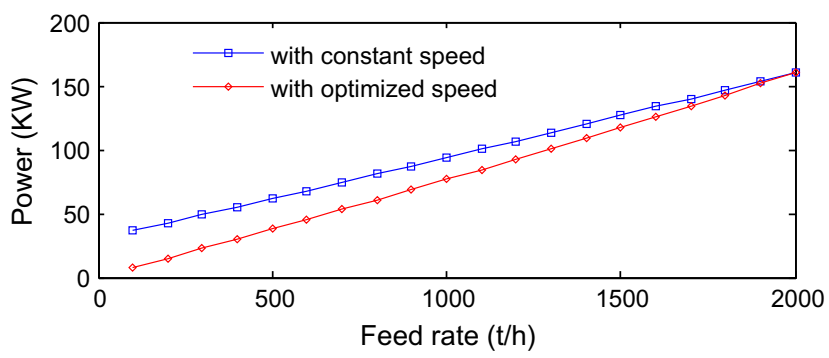

Fig. 7. Energy saving through operation efficiency optimization. in Fig. 8. Hence, load shifting of the belt conveyor is also achieved through the operation efficiency optimization. Problem three does not deal with the ramp rate of belt speed, hence, large changes happen to both the belt speed and feed rate. It harms certain equipment or components of belt conveyors. Problem four involves ramp rate of belt speed into objective for minimization, consequently, the belt speed and feed rate in this problem are much smoother than those in problem three. Moreover, the weight $\varpi$ affects the ramp rate in such a way that a larger $\varpi$ results in smoother belt speed and feed rate, as shown in Fig. 8.

As shown in Table 1, transferring the same amount of material within one day, optimization problem two consumes the least energy, however, it results in the highest energy cost because the TOU tariff is not considered by this optimization problem. Optimization problem four consumes more energy, consequently, results in more cost than problem three. However, this sacrifice is compensated by the improvement of the profiles of the belt speed and feed rate. As can be seen, integrating a technical constraint into the objective function can indeed balance an economic indicator and a technical indicator.

Finally, optimization problem five and six are simulated. The two problems are applicable for the cases, where the feed rate is uncontrollable but predictable. The feed rate forecast as shown in Fig. 9 is used for simulation. Optimization problem five regulates the belt speed to suit the feed rate. Optimization problem six originates from problem five, however, it deals with the ramp rate of belt speed by adding $\varpi \sum_{j=1}^{N-1}\left(V_{j+1}-V_{j}\right)^{2}$ to the objective for minimization. The smooth belt speed is obtained by problem six as shown in Fig. 9. Again, a larger $\varpi$ results in smoother belt speed and more energy consumption.

\subsection{Remark on muti-objective optimization}

The above six optimization problems optimize the energy efficiency of belt conveyors with different objectives along with different constraints. Each of them employs a single objective for optimization. However, the energy efficiency of belt conveyor does not exclude muti-objective optimization. In muti-objective optimization, energy consumption, $J_{E}$, and energy cost, $J_{C}$, can be selected as the objectives for minimization subject to relevant constraints. $J_{E}$ always conflicts with $J_{C}$ because of the TOU tariff. However, the muti-objective framework makes it possible to balance the two objectives. The typical constraints of this muti-objective optimization problem may come from belt speed, unit mass of the belt, total product requirement, and the ramp rate of belt speed. All these constraints can be expressed in a general form as $g(V, T) \leqslant 0$. The muti-objective optimization problem can then be formulated as

$\min J_{E}(V, T), \quad J_{C}(V, T)$,

subject to $g(V, T) \leqslant 0$.

In literature, many methods are proposed to solve the mutiobjective optimization problems. The solutions can provide Pareto front information for decision makers. Specifically, the muti-objective optimization of energy efficiency, as shown in (39), has practical usefulness for site managers of belt conveyors to make rational decision with informed tradeoffs. It will be investigated in the future work.

\section{Conclusion}

Belt conveyors are consuming a considerable part of the total energy supply. This paper focuses on the energy saving of belt conveyors through the improvement of operation efficiency, where optimization is employed. We begin with the energy model of belt conveyors which is the base of optimization. The existing energy 

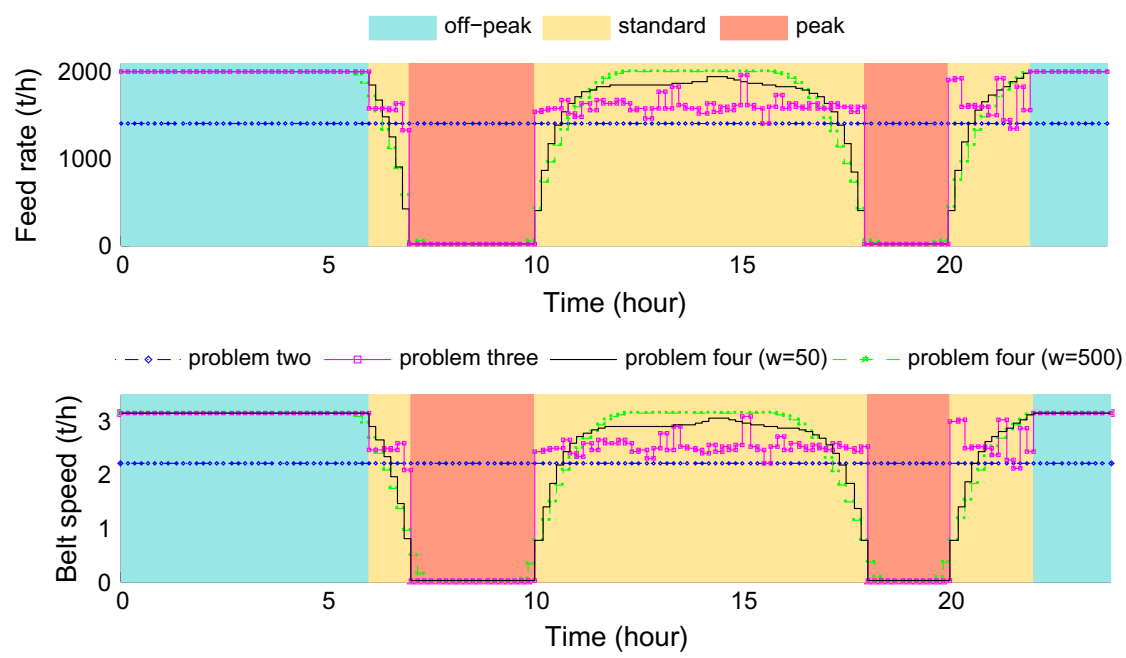

Fig. 8. Operation optimization of problem two, three and four.

Table 1

Energy consumption and energy cost of optimization problem two, three and four $\left(T_{\text {SUM }}=33,600 \mathrm{t}\right)$

\begin{tabular}{lll}
\hline Optimization problems & Energy consumption $(\mathrm{kW} \mathrm{h})$ & Energy cost (Rand) \\
\hline Problem two & 2617.2 & 622.72 \\
Problem three & 2669.7 & 347.23 \\
Problem four $(\varpi=50)$ & 2679.3 & 348.85 \\
Problem four $(\varpi=500)$ & 2684.3 & 352.61 \\
\hline
\end{tabular}
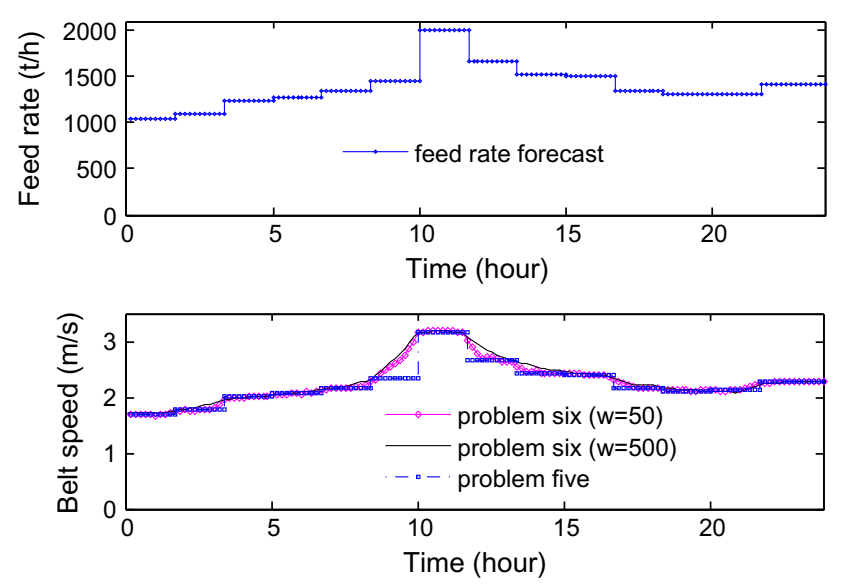

Fig. 9. Operation optimization with predictable feed rate.

models are reviewed and then an analytic model, lumping all the parameters into four coefficients, is proposed. The new model originates from ISO 5048 and is suitable for parameter estimation and optimization. The four coefficients of the new model can be derived from the design parameters or be estimated through field experiments. The latter guarantees an improved accuracy of the model, consequently, the practicability of the energy optimization of belt conveyors.

Off-line parameter estimation, based on LSQ and on-line parameter estimation, based on RLSQ are proposed to the identification of this energy model respectively. Off-line parameter estimation is applicable for the belt conveyors without permanent instruments for electrical power of motor, for belt speed and for feed rate. On the other hand, if a belt conveyor is equipped with permanent instruments, on-line parameter estimate is adopted to update the coefficients of the energy model automatically. Simulation results are presented to show the applicability of the proposed off-line and on-line parameter estimation of the energy model.

Subsequently, energy optimization is put to belt conveyors at the operation level, where the newly proposed model is used. Six optimization problems concerning different aspects of the belt conveyors are proposed and formulated. A typical belt conveyor system is used for simulation. It is shown by simulation that the variable speed control of belt conveyors can indeed save energy. The further a belt conveyor deviates from its design operation condition, the more energy can be saved by speed control. A belt conveyor can be driven in its optimal operation efficiency through the optimization of its performance indicators, e.g., energy consumption or energy cost. With the consideration of TOU tariff, load shifting is achieved by operation efficiency optimization. Further, by integrating a technical issue into the objective function, a balance between the economic indicator and the technical indicator can be obtained.

In this paper, the proposed energy model is used for operation efficiency optimization of a conveying system with a belt conveyor. However, it can indeed be put to practical conveying systems with multiple belt conveyors [35]. The operation efficiency optimization of belt conveyors is formulated as general optimal control problems, hence, various optimization techniques and tools can be used. Further, extra constraints, e.g., the ones from silo capacity or stockpile capacity, can be easily formulated.

\section{References}

[1] Marais J, Mathews E, Pelzer R. Analysing DSM opportunities on mine conveyor systems. In: Industrial and commercial use of energy conference, Cape Town, South Africa; 28-30 May 2008.

[2] Alspaugh MA. Latest developments in belt conveyor technology. In: MINExpo 2004; Las Vegas, NV, USA; 27-30 September 2004.

[3] Xia X, Zhang J, Control systems and energy efficiency from the POET perspective. In: IFAC Conference on Control Methodologies and Technology for Energy Efficiency; Vilamoura, Portugal; 29-31 March, 2010.

[4] Reicks AV. Belt conveyor idler roll behavours. In: Alspaugh MA, editor. Bulk material handling by conveyor belt 7. Colorado: SME; 2008. p. 35-40.

[5] Staniak K, França Jr J. Energy saving in long distance conveyors-Novel idler technology. In: Hennies WT, Ayres da Silva LA, Chaves AP, editors. Mine planning and equipment selection. Rotterdam, Netherlands: Balkema; 1996. p. 479-86.

[6] Tapp AG. Energy saving troughing idler technology. Bulk Solids Handl 2000;20(4):437-49.

[7] Jansen M. The development of energy-optimized conveyor belts - a joint project of the conveyor belt group of ContiTech AG and RWE power AG. World Min 2008;60(2):83-7. 
[8] De Almeida AT, Fonseca P, Bertoldi P. Energy-efficient motor systems in the industrial and in the services sectors in the European Union: characterisation, potentials, barriers and policies. Energy 2003;28:673-90.

[9] Middelberg A, Zhang J, Xia X. An optimal control model for load shifting - with application in the energy management of a colliery. Appl Energy 2009;86:1266-73.

[10] Marx DJL, Calmeyer JE. An integrated conveyor energy model methodology. Trans South African Inst Electr Eng 2004;95:256-64.

[11] Marx DJL, Calmeyer JE. A case study of an integrated conveyor belt model for mining industry. In: IEEE AFRICON 2004; Gaborone, Botswana; 15-17 September, 2004.

[12] Lauhoff $\mathrm{H}$. Speed control on belt conveyors - dose is really save engery? Bulk Solids Handl 2005;25(6):68-77.

[13] Van Den Heuvel B. Investigations dealing with variable-speed drives of belt conveyor systems. World Min 2006;58(2):105-13.

[14] Daus W, Köerber S, Becher N. Raw coal loading and belt conveyor system at Nochten opencast mine - a new conveying and loading system based on drives controlled and adjusted by frequency converter. Braunkohle Surf Min 1998;50(2):117-30.

[15] Köhler U, Sykulla M, Wuschek V. Variable-speed belt conveyors gaining in importance. Braunkohle Surf Min 2001;53(1):65-72.

[16] Koopmann K, Weber D, Keller C, Daus W, Köber S. Conveying device for opencast mines. US Patent 6209711 B1; April 3, 2001.

[17] Steven RB. Belting the worlds' longest single flight conventional overland belt conveyor. Bulk Solids Handl 2008;28(3):172-81.

[18] Gerard B. Optimisation of overland conveyor performance. Aust Bulk Hand Rev 2009:26-36.

[19] ISO. Continuous mechanical handling equipment - belt conveyors with carrying idlers - calculation of operating power and tensile forces, 2 nd ed., ISO $5048 ; 1989$.

[20] DIN. Continuous conveyors-belt conveyors for loose bulk materials - basis for calculation and dimensioning, DIN 22101; 2002
[21] JIS. Rubber belt conveyors with carrying idlers - calculation of operating power and tensile forces, JIS B 8805; 1992.

[22] Hager M, Simonsen H. Calculation and design of belt conveyors for bulk material. Braunkohle Surf Min 2000;52(3):245-60.

[23] GoodYear. Handbook of conveyor and elevator belting. Akron, Ohio: The Goodyear Tire \& Rubber Company; 1975.

[24] Zhang S, Xia X. A new energy calcualtion model of belt conveyor. IEEE AFRICON 2009; Nairobi, Kenya; 23-25 September, 2009.

[25] Songsiri J, Banjerdpongchai D. Dynamic models of servo-driven conveyor system. TENCON 2004; Chiang Mai, Thailand; 21-24 November 2004.

[26] Jeinsh T, Sader M, Ding SX, Engel P, Jahn W, Niemz R. A model-based information system for simulation and mornitoring of belt conveyor systems. Darmstadt, Germany: IFAC Mechatronic System; 2000.

[27] Nuttall AJG, Lodewijks G. Dynamics of multiple drive belt conveyor systems. Part Part Syst Charact 2007;24:365-9.

[28] Ljung L. System identification theory for the user. 2nd ed. New Jersey: Prentice-Hall Inc.; 1999. p. 197-203.

[29] Strejc V. Least squares parameter estimation. Automatica 1980;16:535-50.

[30] Chen HYK, Postel CE. On-line parameter identification of input output curve for thermal units. IEEE Trans. Power Syst. 1986;PWRS-1(2):221-4.

[31] Hsia TC. On least squares algorithms for system parameter identification. IEEE Trans. Autom. Control 1976:104-8.

[32] Koubaa Y. Recursive identification of induction motor parameters. Simul Modell Pract Theory 2004;12:363-81.

[33] Alonge F, D'Ippolito F, Raimondi FM. Least squares and genetic algorithms for parameter identification of induction motors. Control Eng Pract 2001;9:647-57.

[34] Spaans C. The calculation of the main resistance of belt conveyors. Bulk Solids Handl 1991;11(4):809-20.

[35] Zhang S, Xia X. Optimal control of operation efficiency of belt conveyor systems. Appl Energy 2010. doi:10.1016/j.apenergy.2010.01.006). 